\title{
A computational neuron model based on Poisson-Nernst-Planck theory
}

\author{
P. M. Nanninga ${ }^{1}$
}

(Received 1 August 2008; revised 17 September 2008)

\begin{abstract}
Modelling of the nerve impulse is often simplified to one spatial dimension, for example by using cable theory. In reality signals propagate in a complex three dimensional environment, and neurons may electrostatically affect cells in close proximity. To investigate this, the electrochemistry of the neuron environment is modelled using the Poisson-Nernst-Planck theory of electrodiffusion. An accurate numerical solver for the Poisson-Nernst-Planck equations in three dimensions is developed. The solver, integrated with a simple computational model of ion channels, is capable of simulating the dynamics of multiple electrical charge species for an arbitrary configuration of membranes and ion channels. Preliminary simulations of simplified neurons show resting membrane potentials broadly consistent with the Goldman equation of electrochemistry, but with interesting differences in some cases. The model can be applied to the detailed study of the nerve impulse.
\end{abstract}

See http://anziamj.austms.org.au/ojs/index.php/ANZIAMJ/article/view/ 1390 for this article, (c) Austral. Mathematical Soc. 2008. Published September 21, 2008. ISSN 1446-8735 


\section{Contents}

1 Introduction

$\mathrm{C} 47$

2 Poisson-Nernst-Planck theory $\quad$ C48

2.1 Poisson-Nernst-Planck equations . . . . . . . . . . . C49

2.2 Poisson-Boltzmann equation . . . . . . . . . . . C50

3 Poisson-Nernst-Planck numerical solver

C51

4 Ion channel model

C54

5 Implementation and results

C56

6 Conclusion

C57

References

C58

\section{Introduction}

The standard explanation for the electric potential observed to occur across neuron cell membranes is the selective transport of ions, mainly through ion channels (Nicholls et al. [7]). In this process of electrodiffusion, the tendency of electric charge to diffuse toward areas of lower concentration (Fick's law), is offset by electric advection (Kohlrausch's law) in which a drift velocity is imparted to charged particles by the electric field of the surrounding ion cloud. When confined to the direction of nerve impulse, the process of one dimensional electrodiffusion may be approximated by cable theory (Qian and Sejnowski [9]) in which the membrane is modelled as a network of electrical resistors, capacitors and batteries. This theory provides the foundation for Hodgkin and Huxley's famous model of the nerve impulse.

This article develops a model of the neuron environment based on the 
Poisson-Nernst-Planck (PNP) theory (Chung et al. [1]) of electrodiffusion, the PNP model. A computational implementation of the PNP model is described, including an accurate numerical solver of the PNP equations. Note that PNP theory is applied here at a scale between that of cable theory (populations of ion channels) and molecular dynamics (individual ion channels).

The PNP computational model has been used for preliminary investigation of the resting potential of neurons. The simulations show non-zero particle fluxes in the steady state, even with a single ion channel, indicating the modelled neuron is not in thermodynamic equilibrium. Results, though broadly consistent with the Goldman equation [3] of electrochemistry, show interesting differences in some cases.

\section{Poisson-Nernst-Planck theory}

The neuron environment comprises densely packed neurons and neuroglia immersed in ionic fluid. The main charge species associated with the membrane potential are $\mathrm{Ca}^{2+}, \mathrm{Cl}^{-}, \mathrm{K}^{+}, \mathrm{Na}^{+}$and organic anions $\mathrm{A}^{-}$.

Owing to their close proximity, neurons should have a direct electrodiffusive effect on their neighbours (Nicholson [8]). For example, in specialized connections such as synapses and gap junctions the intercellular separation may be as little as $3 \mathrm{~nm}$ (nanometres) compared with a more common separation of $25 \mathrm{~nm}$. The ions form a cloud which tends to shield the electrodiffusive effect, and the strength of this shielding depends on factors such as charge concentration (a lower density having a weaker shielding effect) and geometry. A measure of the range of electrostatic effects is the Debye-Hückel screening length $\lambda$. For example, in the case of $\mathrm{K}^{+}$ions at a concentration of $3 \mathrm{mM} / \mathrm{l}$ (millimoles/litre) in mammal extracellular fluid, the screening length $\lambda_{\mathrm{K}^{+}} \approx 8.1 \mathrm{~nm}$.

To investigate electrodiffusion in the full 3D neural environment, Poisson- 
Nernst-Planck (PNP) theory is adopted. This theory is based on the fundamental physical laws of mass and charge conservation. Charge conservation is formalized through the Poisson equation and mass conservation through a set of transport equations, one for each charge species. Fick's and Kohlrausch's laws are incorporated into the transport equations. PNP theory assumes a continuum, so that, for example, charge concentrations are assumed to be (piecewise) continuous distributions. Within the neuron environment discontinuities occur where fluid meets cell membrane. In the standard meanfield approximation, interactions between mobile charges are ignored (this assumption must break down for sufficiently high ion concentrations), so that PNP theory is essentially electrostatic.

Mathematically, PNP theory is formalized through a set of nonlinear second order partial differential equations (elliptic and parabolic) which must be solved self-consistently with the boundary conditions of the system being considered. Analytic solution of the PNP equations is a topic in itself and I investigate this in detail elsewhere.

\subsection{Poisson-Nernst-Planck equations}

Consider a continuum of fixed and mobile electric charges. Neglecting nonelectrostatic effects, the electric field $\vec{E}=-\nabla \phi$ where the (electrostatic) potential $\phi(\vec{r}, t)$ at location $\vec{r}$ and time $t$ satisfies the Poisson equation

$$
\nabla \cdot(\epsilon \nabla \phi)=-\rho_{\mathrm{f}}-\mathrm{F} \sum_{i=1}^{\mathrm{N}} v_{i} \mathrm{c}_{i} .
$$

In this equation, $\epsilon(\vec{r}, t)$ is the electric permittivity of the medium, $\rho_{f}(\vec{r}, t)$ is the density of fixed charges, $\mathrm{F}$ is the Faraday constant, $v_{i} \neq 0$ is the valence of the ith of $N$ mobile charge species with $c_{i}(\vec{r}, t)$ the molar concentration.

Mass conservation requires the rate of change of a charge species' concentration to balance the divergence of its flux. This gives one transport 
equation for each mobile charge species: $\partial\left(N_{A} c_{i}\right) / \partial t+\nabla \cdot \vec{j}_{i}=0$, where $N_{A}$ is Avogadro's number and $\vec{j}_{i}:=-D_{i}\left\{\left[N_{A} c_{i} /(k T)\right] \nabla V_{i}+\nabla\left(N_{A} c_{i}\right)\right\}$ is the particle flux vector for the $i$ th mobile charge species. Here $D_{i}(\vec{r})$ is the diffusion coefficient (diffusivity), $k$ is Boltzmann's constant, $T$ is the temperature, and $V_{i}(\vec{r}, t):=U_{i}+q_{e} v_{i} \phi$ is the potential energy. In this last equation $\mathrm{q}_{e}$ is the magnitude of the electron charge and $\mathrm{U}_{i}$ is the potential energy due to non-electrostatic interactions.

Again assuming electrostatic interactions dominate, set $U_{i}=0$ so that the flux $\vec{j}_{i}=-D_{i} N_{A}\left(\xi \nu_{i} c_{i} \nabla \phi+\nabla c_{i}\right)$, where the constant $\xi:=q_{e} /(k T)$. The first term in this expression for the particle flux represents the contribution from electric advection, and the second term represents flux due to diffusion. The transport equations become

$$
\frac{\partial c_{i}}{\partial t}=\nabla \cdot\left[D_{i}\left(\xi \nu_{i} c_{i} \nabla \phi+\nabla c_{i}\right)\right] .
$$

The coupled system (1) and (2) are the PNP equations.

\subsection{Poisson-Boltzmann equation}

In a steady state the concentrations do not change with time: $\partial c_{i} / \partial t=0$. If also the particle flux $\vec{j}_{i}=\overrightarrow{0}$ for all charge species, then the transport equations are all trivially satisfied. The vanishing of the particle fluxes requires $\xi \nu_{i} c_{i} \nabla \phi+\nabla c_{i}=\overrightarrow{0}$ for all $i$, independent of diffusivities, which integrates to $c_{i}=c_{i, 0} e^{-\xi v_{i}\left(\phi-\phi_{0}\right)}$. This is a form of the Nernst equation (with concentrations instead of chemical activities, the dilute concentrations assumption). Substituting $c_{i}$ from the Nernst equation into the Poisson equation (1), gives the nonlinear Poisson-Boltzmann (PB) equation

$$
\nabla \cdot(\epsilon \nabla \phi)+\mathrm{F} \sum_{i=1}^{\mathrm{N}} \nu_{i} c_{i, 0} e^{-\xi \nu_{i}\left(\phi-\phi_{0}\right)}=-\rho_{\mathrm{f}} .
$$

For given boundary conditions, the solution to the PB-equation is unique. 


\section{Poisson-Nernst-Planck numerical solver}

The core of the PNP computational model is a numerical solver of the PNP equations. Kurnikova et al. [6] considered the PNP equations in the context of an individual ion channel. However, their focus, like many others, has been the steady state rather than dynamics. They transformed the steady state transport equations and then solved the transformed system numerically using a successive over-relaxation method. To study the nerve impulse, dynamics are of central interest, and so the full PNP equations must be solved.

Consider a 2D system (readily extended to 3D) modelled using a square grid of side $h$ with rows labelled $i=1, \ldots, m+1$ and columns $j=1, \ldots, n+$ 1. Taking finite differences of the Poisson equation (1) at the edges of the grid cell centred at the computational point $i, j$, and letting the total charge density (fixed plus mobile) be $\rho$, then

$$
\left(\epsilon_{i, j}^{1} \phi_{i+1, j}+\epsilon_{i, j}^{2} \phi_{i-1, j}+\epsilon_{i, j}^{3} \phi_{i, j+1}+\epsilon_{i, j}^{4} \phi_{i, j-1}-\epsilon_{i, j}^{0} \phi_{i, j}\right) \sim-h^{2} \rho_{i, j}
$$

in which $\epsilon_{i, j}^{1}:=\epsilon_{i+\frac{1}{2}, j}, \epsilon_{i, j}^{2}:=\epsilon_{i-\frac{1}{2}, j}, \epsilon_{i, j}^{3}:=\epsilon_{i, j+\frac{1}{2}}, \epsilon_{i, j}^{4}:=\epsilon_{i, j-\frac{1}{2}}$ and $\epsilon_{i, j}^{0}:=\sum_{k=1}^{4} \epsilon_{i, j}^{k}$. To get (4) the partial derivatives of the electric potential evaluated at cell edges were approximated as $\left(\phi_{x}\right)_{i, j+\frac{1}{2}} \sim\left(\phi_{i, j+1}-\phi_{i, j}\right) / h$, $\left(\phi_{x}\right)_{i, j-\frac{1}{2}} \sim\left(\phi_{i, j}-\phi_{i, j-1}\right) / h,\left(\phi_{y}\right)_{i+\frac{1}{2}, j} \sim\left(\phi_{i+1, j}-\phi_{i, j}\right) / h$, and $\left(\phi_{y}\right)_{i-\frac{1}{2}, j} \sim$ $\left(\phi_{i, j}-\phi_{i-1, j}\right) / h$. The electric permittivity at cell edges was approximated with an arithmetic mean, for example $\epsilon_{i, j}^{3} \sim \frac{1}{2}\left(\epsilon_{i, j}+\epsilon_{i, j+1}\right)$, although a harmonic mean may give better accuracy at discontinuities. With these approximations, equation (4) becomes second order accurate in space.

At a dielectric discontinuity, such as the boundary between a membrane and ionic fluid, continuity of the normal component of the electric displacement vector (Visscher [10]) leads to the condition $\epsilon_{(-)} \vec{n} \cdot \nabla \phi_{(-)}=\epsilon_{(+)} \vec{n}$. $\nabla \phi_{(+)}$, where -, + denote the different dielectrics on either side of the discontinuity and $\vec{n}$ is the normal vector to the interface. For example, at the computational point $i, j$, assuming this was the location of a vertical discontinuity, the continuity condition is approximated by $\epsilon_{i, j_{-}}\left(\phi_{x}\right)_{i, j_{-}}=\epsilon_{i, j_{+}}\left(\phi_{x}\right)_{i, j_{+}}$. 
Using this with a finite volume approach it is demonstrated that the finite differencing scheme retains second order accuracy at discontinuities.

Assuming Dirichlet boundary conditions, the set of equations (4), one equation for every interior computational point in the system (there are a total of $k:=(m-1)(n-1)$ interior points), may be expressed in matrix form as $\mathbf{A} \vec{\phi}=\vec{f}$ where $\mathbf{A}$ is a $k \times k$ matrix of electric permittivity values, $\vec{\phi}$ is a column vector with $k$ elements which are the values $\phi_{i, j}$ listed in row (lexicographic) order, and $\vec{f}$ is is a column vector with $k$ elements which are known (boundary) values in the difference equations. The matrix $\mathbf{A}$ is always sparse and symmetric (in 2D this is because $\epsilon_{i, j}^{3}=\epsilon_{i, j+1}^{4}$ ). In 2D $\mathbf{A}$ is block tridiagonal with two fringe diagonals (five diagonals in all). In $3 \mathrm{D} \mathbf{A}$ is again block tridiagonal but with two flanking pairs of fringe diagonals (so seven diagonals in all). When $\mathbf{A}$ is positive definite (which it usually is), the linear system $\mathbf{A} \overrightarrow{\boldsymbol{\phi}}=\vec{f}$ is solved directly using, for example, some form of conjugate gradient method. Alternatively, an approximate solution is obtained through an iterative method.

The transport equation for the ith mobile charge species is given by (2). To avoid confusion with subscripts identifying grid cells, in what follows the subscript for the ith mobile charge species will be dropped, so that $\mathrm{c}$ will be used to represent any concentration $c_{i}$, with valence $v=v_{i}$, etc.

The transport equation includes a time derivative term which is approximated with a single level forward time method. Denoting evaluation at the $n$th time level by a superscript $(n)$, and evaluating the derivative at time level $n+\frac{1}{2}$, then $(\partial c / \partial t)_{i, j}^{\left(n+\frac{1}{2}\right)} \sim\left(c_{i, j}^{(n+1)}-c_{i, j}^{(n)}\right) / p$, where $p$ is the time-step (independent of $n$ ) between levels $n$ and $n+1$. The right side of the transport equation is differenced in the same way as the Poisson equation. For computational point $i, j$ at time level $n$, this centred differencing gives

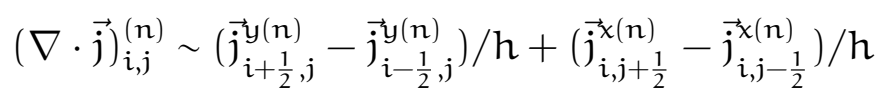

where, for example, $\vec{j}_{\mathfrak{i}+\frac{1}{2}, j}^{(\mathfrak{n})}$ is the component of the vector flux in the $y$ - 
direction at time level $n$ through the side $\left(\right.$ at $i+\frac{1}{2}$ ) of the grid cell centred at $i, j$. Particle fluxes through the four sides of this cell are approximated by

$$
\begin{aligned}
& \vec{j}_{i \pm \frac{1}{2}, j}^{y(n)} \sim-D_{i \pm \frac{1}{2}, j}^{(n)}\left(\frac{1}{k T} c_{i \pm \frac{1}{2}, j}^{(n)}\left(V_{x}\right)_{i \pm \frac{1}{2}, j}^{(n)}+\left(c_{x}\right)_{i \pm \frac{1}{2}, j}^{(n)}\right), \\
& \vec{j}_{i, j \pm \frac{1}{2}}^{x(n)} \sim-D_{i, j \pm \frac{1}{2}}^{(n)}\left(\frac{1}{k T} c_{i, j \pm \frac{1}{2}}^{(n)}\left(V_{x}\right)_{i, j \pm \frac{1}{2}}^{(n)}+\left(c_{x}\right)_{i, j \pm \frac{1}{2}}^{(n)}\right) .
\end{aligned}
$$

Here $V:=q_{e} v \phi$ is the potential energy of the ith mobile charge species (ignoring non-electrostatic interactions). Note that the diffusion coefficients have been indexed by $n$ to allow for changes in their values with time.

The partial derivatives in (6) are approximated in the same way as the partial derivatives of the electric potential in the Poisson equation. Assume the values of the concentration at the edges of the grid cells are approximated by an arithmetic mean, for example $c_{i, j+\frac{1}{2}} \sim\left(c_{i, j}+c_{i, j+1}\right) / 2$.

The notation introduced earlier is extended to diffusivity values $D_{i, j}^{1}:=$ $D_{i+\frac{1}{2}, j}, D_{i, j}^{2}:=D_{i-\frac{1}{2}, j}, D_{i, j}^{3}:=D_{i, j+\frac{1}{2}}$, and $D_{i, j}^{4}:=D_{i, j-\frac{1}{2}}$. Similarly for the concentrations $c_{i, j}^{0}:=c_{i, j}, c_{i, j}^{1}:=c_{i+1, j}, c_{i, j}^{2}:=c_{i-1, j}, c_{i, j}^{3}:=c_{i, j+1}$, and $c_{i, j}^{4}:=c_{i, j-1}$. The notation for the concentrations applies in exactly the same way to the potential energy $\mathrm{V}$. Also, it is convenient to define $\eta_{i, j}^{k(n)}:=1+\left(V_{i, j}^{k(n)}-V_{i, j}^{o(n)}\right) /(2 k T)$ and $\psi_{i, j}^{k(n)}:=1-\left(V_{i, j}^{k(n)}-V_{i, j}^{o(n)}\right) /(2 k T)$. Then the fluxes in (6) are written compactly as

$$
\begin{aligned}
& \vec{j}_{i+\frac{1}{2}, j}^{y(n)} \sim-D_{i, j}^{1(n)}\left(-c_{i, j}^{o(n)} \psi_{i, j}^{1(n)}+c_{i, j}^{1(n)} \eta_{i, j}^{1(n)}\right) / h, \\
& \vec{j}_{i-\frac{1}{2}, j}^{((n)} \sim-D_{i, j}^{2(n)}\left(c_{i, j}^{o(n)} \psi_{i, j}^{2(n)}-c_{i, j}^{2(n)} \eta_{i, j}^{2(n)}\right) / h, \\
& \vec{j}_{i, j+\frac{1}{2}}^{x(n)} \sim-D_{i, j}^{3(n)}\left(-c_{i, j}^{o(n)} \psi_{i, j}^{3(n)}+c_{i, j}^{3(n)} \eta_{i, j}^{3(n)}\right) / h, \\
& \vec{j}_{i, j-\frac{1}{2}}^{x(n)} \sim-D_{i, j}^{4(n)}\left(c_{i, j}^{o(n)} \psi_{i, j}^{4(n)}-c_{i, j}^{4(n)} \eta_{i, j}^{4(n)}\right) / h .
\end{aligned}
$$

In the backward Euler or backward time approximation, time level $n+1$ is used instead of time level $n$. A more accurate approximation than either 
the forward or backward time methods, is to use the average of both. This is the Crank-Nicolson method (Crank and Nicolson [2]), and it is second order accurate in time and space. Adopting the Crank-Nicolson method to approximate the flux divergence, the transport equation becomes

$$
\left(c_{i, j}^{(n+1)}-c_{i, j}^{(n)}\right) / p \sim-\left[(\nabla \cdot \vec{j})_{i, j}^{(n)}+(\nabla \cdot \vec{j})_{i, j}^{(n+1)}\right] / 2 .
$$

Now use (7) in (5) and then use the result in (8). Collecting time level $n+1$ terms on the left and time level $n$ terms on the right, then gives

$$
c_{i, j}^{o(n+1)} \alpha_{i, j}^{(n+1)}-\sum_{k=1}^{4} c_{i, j}^{k(n+1)} \beta_{i, j}^{k(n+1)} \sim c_{i, j}^{o(n)} \gamma_{i, j}^{(n)}+\sum_{k=1}^{4} c_{i, j}^{k(n)} \beta_{i, j}^{k(n)}
$$

where $\alpha_{i, j}^{(\mathfrak{n})}:=1+\lambda \sum_{k=1}^{4} D_{i, j}^{k(n)} \psi_{i, j}^{k(n)}, \beta_{i, j}^{k(n)}:=\lambda D_{i, j}^{k(n)} \eta_{i, j}^{k(n)}$ for $k=1, \ldots, 4$, $\gamma_{i, j}^{(n)}:=1-\lambda \sum_{k=1}^{4} D_{i, j}^{k(n)} \psi_{i, j}^{k(n)}$, and $\lambda:=p /\left(2 h^{2}\right)$. The extension from a $2 D$ grid to a $3 \mathrm{D}$ (hexahedral) grid is now simple: the index $k$ in (9) runs to six instead of four. The above differencing scheme is readily adapted to a non-uniform discretization, if required. Other methods to solve advectiondiffusion-reaction equations are given by Hundsdorfer [5].

As with the discretized Poisson equation, (9) is readily formulated as a sparse linear system, allowing concentrations to be computed from their values at the previous time step, and the values of the electrostatic potential. The newly computed concentrations are then used to update the electric potential values by solving the linear system $\mathbf{A} \vec{\phi}=\vec{f}$. In this way the coupled PNP equations are solved numerically. Testing against analytic solutions shows that the scheme works accurately.

\section{Ion channel model}

Ion channels are central to the standard explanation of membrane potentials and the nerve impulse (Hille [4]). When an ion passes close to an ion channel 
protein molecule, there will be discrete charge effects from individual atoms in close proximity to the ion, and the protein molecule itself is subject to complex conformational changes. These and other effects lead to the detailed operation of an ion channel. To understand the operation of ion channels, an approach such as molecular dynamics is useful (Chung et al. [1]), although PNP theory has been applied with success to the modelling of individual ion channels (Chung et al. [1], Kurnikova et al. [6]).

In the PNP computational model the ion channel is simply a narrow passage through the membrane. Grid cell elements in the model are tagged with attributes identifying them as belonging to intracellular or extracellular fluid, lipid membrane, ion channel protein, ion channel fluid, etc. The type of ion channel is also tagged, for example passive $\mathrm{K}^{+}$channel, voltage-gated $\mathrm{Na}^{+}$channel, and ligand-activated channel. The electric permittivities and diffusivities are permitted to take on different values according to the cell tag values.

The operation of an ion channel is explicitly coded into the model. For example, the fluid inside a $\mathrm{K}^{+}$ion channel acts as an impermeable barrier to any ions other than $\mathrm{K}^{+}$. For $\mathrm{K}^{+}$ions the fluid inside a passive channel acts like extracellular or intracellular ionic fluid and is subject to the usual drivers of diffusion and electric advection. The situation is different for a voltagegated $\mathrm{K}^{+}$channel because it is tagged with a voltage threshold value. If the local membrane potential exceeds this threshold, then the channel fluid acts as ionic fluid and the ions may pass; but if the threshold is not exceeded, then the channel will act as an impermeable barrier. For a ligand-activated channel, the presence of at least a threshold level of ligand concentration is required to (selectively) open the channel. A similar approach may be taken to the modelling of ion pumps, synapses, etc. 


\section{$5 \quad$ Implementation and results}

The PNP computational model has been implemented using MATLAB since it offers efficient solution of large sparse linear systems, and provides useful graphing, plotting and other facilities. The model has been validated against several analytic solutions of the PNP equations and gives accurate results. For example, for a uniform fluid of five mobile charge species subject to an external applied voltage the model converges smoothly to the analytic (PB) solution with negligible error, independent of diffusivities.

Although the model validates well against analytic solutions and is reasonably computationally efficient, testing its fidelity as a biological model requires further work. At this stage simplified neurons with only one or two ion channels have been simulated, but with realistic values for biological parameters such as membrane thickness, electric permittivity, ion concentrations and diffusivities. The results verify the standard model as far as the basic generation of an equilibrium potential is concerned. For example, with a grid cell size of $1 \mathrm{~nm}$ and time-step of $0.1 \mathrm{~ns}$ a $2 \mathrm{D}$ closed membrane with a single passive $\mathrm{K}^{+}$ion channel and a single passive $\mathrm{Cl}^{-}$channel was modelled. For the initial concentrations of $\mathrm{Ca}^{2+}, \mathrm{Cl}^{-}, \mathrm{K}^{+}, \mathrm{Na}^{+}$and $\mathrm{A}^{-}$ions, the intracellular values assumed were respectively (in $\mathrm{mM} / \mathrm{l}$ ) 10 $0^{-4}, 8,140,15$ and 147, and the initial extracellular concentration values assumed were 1.2, 130, 3, 150 and 25. The computational PNP model produced a steady state membrane potential of approximately $-80 \mathrm{mV}$, which is close to the experimentally observed $\mathrm{K}^{+}$equilibrium potential and a typical resting potential of $-73 \mathrm{mV}$.

Even with a single passive ion channel, the computational PNP model does not give a zero flux steady state, so the system is not in thermodynamic equilibrium and the Nernst equation does not apply. In electrochemistry the Goldman equation, an alternative to the Nernst equation, allows for non-zero particle fluxes, and the membrane potential is then a weighted sum (each weight is the ratio of an ion's permeability to the total permeability) of the 
equilibrium (Nernst) potential for each ion able to permeate the membrane.

According to the Goldman equation the resting potential for a single $\mathrm{K}^{+}$ion channel ought to be the $\mathrm{K}^{+}$equilibrium potential (because the $\mathrm{K}^{+}$permeability is the same as the total permeability). So the Goldman equation assumes zero flux for this case, which is quite different from what the PNP model gives. Such differences arise because of the simplifying assumptions behind the Goldman equation.

The Goldman equation may be derived by assuming that the total current density is zero $\left(\overrightarrow{\mathrm{J}}_{e}:=\mathrm{q}_{e} \sum_{i=1}^{N} v_{i} \overrightarrow{\mathrm{j}}_{i}=\overrightarrow{0}\right)$. The PNP computational model indicates that this assumption holds in the bulk of the fluids (when there is more than one type of ion channel), but breaks down within and near to ion channels (because they only allow specific ion types to pass through, so the total current density cannot be zero there).

\section{Conclusion}

A computational model for the 3D continuum dynamics of electric charges (in the electrostatic approximation) has been developed, with application to the neuron environment (and to the study of semiconductors). The PNP model fills the gap in scale between the very detailed molecular dynamics approach suitable for individual ion channels, and the semi-empirical approach of the cable equation and Hodgkin-Huxley equations. The PNP model allows investigation of the electrical behaviour of neurons and neuroglia in close proximity without assuming idealized geometrical shapes for the structures. Preliminary results with simplified neuron models qualitatively and quantitatively agree with the standard model, but indicate that even with a single type of passive ion channel there is always a particle flux in the steady state, so that the Nernst equation does not apply. The Goldman equation also only approximates the results of the PNP model. 
The permeabilities in the Goldman equation are often derived empirically, so it would be interesting to compare the membrane potential computed using the Goldman equation with the steady state obtained from the computational PNP model for various configurations of ion channels. Other possible applications and extensions of the PNP model include: further investigation of the resting potential; simulation of the nerve impulse; modelling of synapses and gap junctions; and investigation of electrostatic effects of neurons and neuroglia in close proximity.

\section{References}

[1] Chung, S. H., Andersen, O. S. and Krishnamurthy, V., Biological Membrane Ion Channels: Dynamics, Structure and Applications, Springer Science+Business Media, New York, 2007. C48, C55

[2] Crank, J. and Nicolson, P., A practical method for numerical evaluation of solutions of partial differential equations of the heat conduction type, Advances in Computational Mathematics, 6, 207-226, 1996. doi:10.1007/BF02127704 C54

[3] Goldman, D. E., Potential, Impedance, and Rectification in Membranes, Journal of General Physiology, 27, 37-60, 1943. http://www.jgp.org/cgi/reprint/27/1/37 C48

[4] Hille, B., Ion Channels of Excitable Membranes, Sinauer Associates, Sunderland, MA., 2001. C54

[5] Hundsdorfer, W., Numerical Solution of Time-Dependent Advection-Diffusion-Reaction Equations, Springer-Verlag, Berlin, 2003. $\mathrm{C} 54$

[6] Kurnikova, M. G., Coalson, R. D., Graf, P. and Nitzan, A., A Lattice Relaxation Algorithm for Three-Dimensional Poisson-Nernst-Planck 
Theory with Application to Ion Transport through the Gramicidin A Channel, Biophysical Journal, 76, 642-656, 1999. http://www. biophysj.org/cgi/content/full/76/2/642 C51, C55

[7] Nicholls, J. G., Martin, A. R., Wallace B. G. and Fuchs, P. A., From Neuron to Brain, Sinauer Associates, Sunderland, MA., 2001. C47

[8] Nicholson, C., Extracellular space as the pathway for neuron-glial cell interaction, In Kettenmann, H. and Ransom, B.R., editors, Neuroglia, Oxford University Press, New York, 387-397, 1995. C48

[9] Qian, N., and Sejnowski, T. J., Electrodiffusion Model of Electrical Conduction in Neuronal Processes, In Woody, C. D., and McGaugh, J. L., editors, Cellular Mechanisms of Conditioning and Behavioral Plasticity, Plenum Press, New York, 237-244, 1988. C47

[10] Visscher, P.B., Fields and Electrodynamics, John Wiley and sons, USA, 1988. C51

\section{Author address}

1. P. M. Nanninga, Centre for Mathematics and its Applications, Australian National University, Canberra, Australia. mailto:paul.nanninga@anu.edu.au 